\title{
Directive Wavefronts Inside a Time Reversal Electromagnetic Chamber
}

\author{
Houmam Moussa, Andrea Cozza, Michel Cauterman \\ Département de Recherche en Électomagnétisme \\ (CNRS, Supelec-Univ. Paris-Sud) \\ SUPELEC, 3 rue Joliot-Curie, 91192 Gif-sur-Yvette Cedex, France \\ houmam.moussadiss. supelec.fr
}

\begin{abstract}
In this paper, the feasibility of directive wavefronts generation inside a time-reversal electromagnetic chamber (TREC) is investigated in order to propose the TREC system as an alternative EMC test facility. This may be accomplished by applying the time reversal technique on electromagnetic waves inside a reverberation chamber. We evaluate the performance of the TREC in creating directive radiation patterns towards an equipment-under-test (EUT). This evaluation is based on the directivity comparison of a wavefront generated by the TREC, and the one generated by a theoretical model of an antenna array in free space. Numerical simulations of the TREC are in a good agreement with the theoretical array model, showing a root-mean-square (rms) deviation from the mean value of the E-field, over a 3 dB main lobe angle, below $6 \%$.
\end{abstract}

\section{INTRODUCTION}

In the last decades, the use of electronics in the avionic and automotive industries has increased dramatically. These industries are facing the problem of conceiving robust systems with respect to the electromagnetic complex environments encountered nowadays.

Different test methods have been used over the years to verify the radiated-immunity of equipments such as transverse electromagnetic (TEM) and gigahertz TEM cells [1], used for immunity testing with TEM fields. Also, the use of anechoic and semi-anechoic chambers began to spread as a standard method [2]. Currently, reverberation chambers [3] has become available as an alternative test procedure in various standards. The comparison of most interest is generally between reverberation chambers (RCs) and anechoic chambers (ACs) [4], taking into consideration the directivity of the EUT.

Indeed, the evaluation of the shielding effectiveness of electronic equipments may depend on the direction and polarization of the incident field both for testing in RCs and in ACs. For testing in ACs, the main problem is to know whether or not the most critical directions and polarizations have been used because of the limited incoming directions and polarizations employed in typical standard tests.

Otherwise, in RCs all the weaknesses of the EUT are statistically excited at the same time but therefore it is not possible to know which direction of arrival and polarization revealed them. Thus, it is difficult to exploit the test results to improve the EUT shielding effectiveness. Moreover, [5] reveals that testing in an AC may lead to stress levels of electronic components inside the equipment that are 10-15 dB higher than what is achieved in RCs, given the same magnitude of incident field, because of the directional dependance in the coupling measurements to the interior of the EUT.

In a recent paper [6], we proposed an alternative tool which enables the generation of high-intensity coherent wavefronts inside reverberation chambers instead of a high-intensity, statistically homogeneous and isotropic field. The present work extends the properties of this tool, called a Time-Reversal Electromagnetic Chamber (TREC) showed in the fig. 1, to the generation of deterministic high-intensity directive wavefronts towards an EUT. These directive wavefronts can be changed in real-time without modifying the configuration of the system unlike for ACs where we have to move the emitter each time we set a new incoming direction.

The main interest using the TREC system is that we are able, in a deterministic way, to finely test the EUT and say precisely by which direction and polarization a weakness was excited. This insight allows designers to improve their EUT against specific aggressions unlike RCs tests. We take here the advantage of the coherent wavefronts as well as plane-waves in ACs testing, and the high-intensity of the field as in RC.

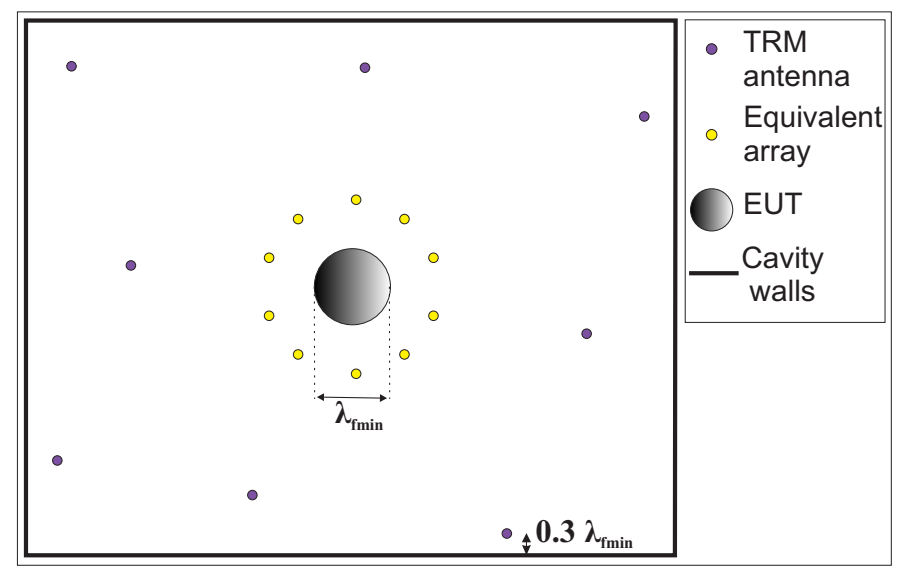

Fig. 1. Time-reversal electromagnetic chamber. The TRM antennas are located at least at $0.3 \lambda_{\text {fmin }}$ from the cavity walls with $\lambda_{\text {fmin }}$ the wavelength corresponding to the minimal frequency of an excitation signal.

This may be accomplished through the use of time-reversal technique [7] on electromagnetic waves inside RCs. These aspects will be developed in the following sections. 
To validate this property, we simulated the TREC system in 2D for a basic comprehension of the mechanisms involved when time reversal is applied inside a reverberation chamber. In fact, this case is rather a simplified 3D than a pure 2D one as only one dimension of the cavity is reduced below the wavelength of use. It provides a realistic testbed for the properties of the time-reversal technique as applied in a highly reverberating cavity, although not as complex as for a fully $3 \mathrm{D}$ structure because we are only dealing with a scalar field with just one vertical polarization. This allowed us to study different configurations of this system as detailed in [6].

\section{TIME REVERSAL FOR EMC TESTS}

The time reversal is a time-domain technique used for many years in acoustics and more recently in electromagnetism [8], to focus a wave on point-like transceivers with respect to the wavelength. In the case of electromagnetic field, these transceivers are simply antennas. The principle of time reversal is to let acoustic or electromagnetic waves live backward their propagation in a complex environment to focus back to the initial point of their generation.

To achieve that, time reversal requires two steps: first, the field must be sampled in every direction by a receivers array, disposed on an area enclosing the source of the field, called a time-reversal mirror (TRM) [7]. To be precise, the electric field $\mathrm{e}\left(\overrightarrow{\rho_{i}}, \mathrm{t}\right)$ detected by a set of antennas located at positions $\overrightarrow{\rho_{i}}$, is sampled during a time interval $\Delta \mathrm{T}$. Then, in a second step, the field is retransmitted by the same antennas in a reversed temporal chronology (last in, first out) equivalent to the transmission of $\mathrm{e}\left(\overrightarrow{\rho_{i}}, \Delta \mathrm{T}-\mathrm{t}\right)$. This procedure allows to convert a divergent wave issued from an electromagnetic source into a convergent wave focusing on this source. Also, in [9], it has been proven that in a strongly reverberating environment, like a reverberation chamber, the number of antennas needed for the TRM can be reduced to a single one instead of a whole set. In fact, for a sufficiently long sampling time $\Delta \mathrm{T}$, all information about the radiated field will be gathered by the reflections into this antenna.

Nevertheless, in EMC tests, EUTs can be electrically large systems that cannot be considered as an antenna and in which excitation signals cannot be injected through a port. Thus, we are not able to obtain directly the time-reversal first-step signals required to produce a focusing wavefront on an EUT during the second step. To overcome this issue, we invoked the field equivalence principle [10] to create a radiation pattern around the EUT. The idea is to define a boundary surface around the EUT on which equivalent electromagnetic currents radiate the desired pattern. These currents are related to the tangential components of the fields on the surface. Besides, for a 2D configuration this surface becomes a line so, in [6], we implemented it with a circular array of isotropic elements, that we called equivalent array in Figure 1, to produce a cylindrical wavefront around the EUT.

We can notice that the rectangular cavity of the TREC system constitutes a propagation channel between the equivalent array and the set of dipole antennas used for the TRM. Indeed, the cavity shows a "discrete" spectrum corresponding to its resonance modes which allows the transmission of the energy inside it. Thus, it is necessary to feed the equivalent array with a signal that will excite a sufficient number of modes, i.e., the frequency and the bandwidth of the excitation signal $\mathrm{x}(t)$ must be chosen according to the associated modal density which can be estimated through Weyl's formula [11]. Moreover, the EUT can be considered as a part of the cavity because it is static inside it during the two steps of time reversal. That is why, its effect on the cavity modes, i.e., the propagation channel, is already taken into account unlike the case of RCs where it is hard to predict, a priori, the effects of a large EUT after its introduction into the chamber.

Another important point is that as we want to illuminate the EUT with a convergent wavefront we need a radiation pattern outward the EUT as it is the case when an antenna radiates a divergent wavefront during the first step of time reversal. The fact is that the circular array alone will radiate both outward and towards the EUT because it is composed of isotropic elements. In order to avoid the radiation of energy towards the EUT, we added two circular row to the initial one, each one spaced by $\lambda_{f c} / 4$ and phase-shifted by one radiant, to obtain a constructive interference for the field away from the EUT and a destructive one towards it, based on the principle of end-fire arrays ( $\lambda_{f c}$ is computed for the central frequency of $x(t)$ ).

An example of an impinging cylindrical wavefront generated by the TREC system is given in figure 2 .

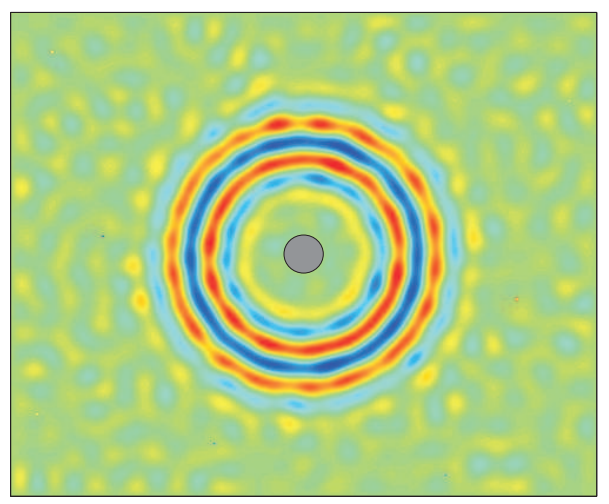

Fig. 2. Circular wavefront impinging on an EUT located at the center of the TREC. 8 antennas were used for the TRM.

We are using a Gaussian pulse-modulated signal, $\mathrm{x}(t)$, so by knowing its time length $\Delta t$ we also know its spatial length $\Delta s$ as $\Delta s=\mathrm{c}_{0} \Delta \mathrm{t}$, where $\mathrm{c}_{0}$ is the wave propagation speed inside the cavity. Thus, if we want to observe the whole pulse before it interacts with the cavity walls, we must take at least $4 \Delta s$ for the smallest dimension of the cavity. During a temporal window, before the wavefront reaches the walls, the wave will propagate in the same conditions as in free space. As a result, we assumed that the equivalent array could be designed using free-space synthesis rules. Afterward, it is the duty of the time-reversal technique to reproduce backward this temporal window, creating a coherent wavefront converging towards 
the EUT after a transient of incoherent field distribution. It is interesting to notice that this distribution shares the same properties of the classical $\mathrm{RC}$ one.

To have a reference for the pattern emitted by the equivalent array, we used a simple free-space 2D model for the radiation of the isotropic sources, expressed in [12] for the frequency domain, as follows:

$$
E(\vec{d}, \omega)=X(\omega) \sum_{i=1}^{N} K_{0}\left(j k\left(\left\|\vec{d}-\overrightarrow{d_{i}}\right\|\right)\right) \exp j \phi_{i}
$$

Here $\mathrm{E}(\vec{d}, \omega)$ is the electric field value at a position $\vec{d}$ while $\overrightarrow{d_{i}}$ represents the location of each isotropic source of the equivalent array. $\omega$ is a given pulsation, $K_{0}$ is the modified second-kind Bessel function of zero-order and $k=\omega / \mathrm{c}_{0}$ is the angular wave number. $\mathrm{X}(\omega)$ is the excitation signal applied on the array and $\phi_{i}$ is the phase associated to each element of the equivalent array circular rows. Finally once we have mapped the field inside a rectangular domain corresponding to the cavity dimensions by the use of equation (1), we observe it in time by means of an inverse Fourier transform.

The distance between elements for each row should be carefully chosen. Inside RCs the spatial correlation functions must be taken into account to have a discrete distribution of the equivalent electromagnetic currents. The spatial correlation between two antennas, omnidirectional in their azimuth plan, have been estimated in [13] to be $\mathrm{J}_{0}\left(k\left(\left\|\overrightarrow{d_{1}}-\overrightarrow{d_{2}}\right\|\right)\right)$ inside $2 \mathrm{D}$ cavities, where $\mathrm{J}_{0}$ is the first-kind Bessel function of zeroorder, and $\overrightarrow{d_{1}}, \overrightarrow{d_{2}}$ two positions in the cavity. As a consequence, we spaced the elements of the circular rows by at most $\lambda_{f c} / 4$ to be able to rebuild properly the distribution on the entire surface.

On the contrary, if we look now at the TRM antenna's positions, they should be placed sufficiently far from each other to record uncorrelated signals that give us complementary information on the the field.

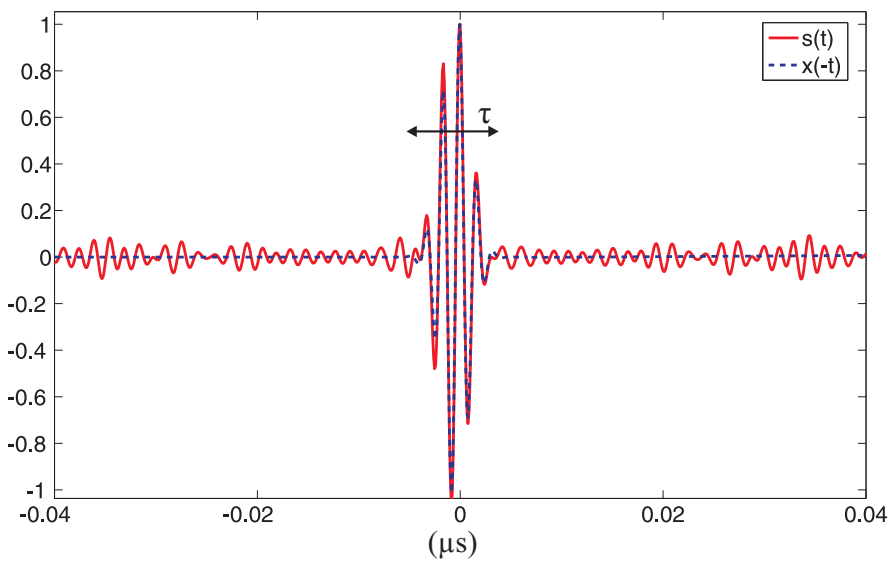

Fig. 3. Temporal-reconstructed signal $\mathrm{s}(\mathrm{t})$ of a pulse-modulated excitation signal $\mathrm{x}(\mathrm{t})$. The signals are normalized to their respective maxima and $\tau$ is the length of $\mathrm{x}(\mathrm{t})$.

As we previously said, the elements of the equivalent array are fed with a pulse-modulated signal $\mathrm{x}(t)$. This signal gives rise to a wavefront which propagates inside the cavity and is sampled by the TRM antennas during a period $\Delta T$. Then the signals $\mathrm{y}_{i}(t)$, obtained on each TRM antenna after this period, are time-reversed and emitted again through the TRM. Finally, after the same time length $\Delta T$, we obtained a signal $\mathrm{s}_{j}(t)$ of the refocused pulse on the equivalent array elements apiece. We observe in Figure 3 the comparison between the excitation signal reversed in time $\mathrm{x}(-t)$ and the signal $\mathrm{s}(t)$ for one element of the equivalent array. We can see that the shape of the pulse is preserved while ringing artifacts appear on the sides. Again, the cavity shows a "discrete" spectrum that filters the continuous spectrum of $\mathrm{x}(t)$ and introduces a degradation on the signal while it propagates. This is the same principle as for the Gibbs phenomenon for a square wave when the number of terms of its Fourier series expansion is truncated leading to these ringing artifacts.

From now on, we will name "noise" the residual error on a reconstructed signal or wavefront, due to the fact that we have a limited number of modes excited inside the cavity. To reduce this noise we need to be in the over-moded range of the cavity. As well, the number of available modes is the number of excited modes by a set of antennas, like an "effective modal density", rather than the estimated modal density over a bandwidth. For instance, we have shown in [6] that the quality of a reconstructed cylindrical wavefront changes with the number of antennas used for the TRM and their different locations.

To quantify the quality of a refocused signal $s(t)$ and thus set the parameters of $\mathrm{x}(t)$, we use the parameter $\gamma$ introduced in [14]. It defines a signal to noise ratio (SNR) between the squared level of the peak of $\mathrm{s}(t), I_{\text {peak }}=s^{2}(0)$, and the level of the noise, $I_{\text {noise }}$, defined as the square of the $\mathrm{s}(t)$ 's rms computed apart from the coherent portion of the reconstructed signal time length $\tau$.

It has been proved in [14] that, for ensemble averages, $\gamma$ depends on parameters resumed in the (2).

$$
\gamma=\frac{\langle s(0)\rangle^{2}}{\left\langle\overline{s^{2}(t)}\right\rangle} \simeq \frac{4 \sqrt{\pi} n_{0} \Delta \omega}{1+2 \pi n_{0} / \Delta T}
$$

These are : the modal density of the cavity, $n_{0}$, considered constant over the bandwidth $\Delta \omega$ of $\mathrm{x}(t)$ and the length $\Delta T$ of the signal recorded by the TRM during the first step of time reversal. Thus, the SNR $\gamma$ corresponding to the signal of Figure 3 is $26 \mathrm{~dB}$ using directly the ratio $I_{\text {peak }}$ over $I_{\text {noise }}$ while the estimation with (2) expects $25.4 \mathrm{~dB}$. Therefore, in an experimental setup, the expected performances would be close to $42.7 \mathrm{~dB}$ as the recording time $\Delta T$ can be considered infinite compared to the simulation one.

\section{DiRECTIVITY INSIDE TREC}

We are interested in extending the properties of the TREC system by generating deterministic directive wavefronts impinging over an EUT. In [6], we have demonstrated the ability of this system to generate a cylindrical wavefront by applying the required signals on the TRM antennas. As explained 
before, these signals were obtained by sampling the field radiated by the equivalent array with the TRM during the first step of time reversal.

Nevertheless, we analyzed them in the frequency domain which is more suited for transfer functions analysis, as shown in (3):

$$
Y_{i}(\omega)=X(\omega) \sum_{j=1}^{N} \alpha_{j} H_{i j}(\omega)
$$

where $Y_{i}(\omega)$ corresponds to the signal received by the $i$-th antenna of the TRM, $X(\omega)$ is the spectrum of the excitation signal that would be applied on each antenna of the equivalent array, and $\alpha_{j}$ is the associated weight to the $j$-th equivalent array element whose function will be clarified later on. $H_{i j}(\omega)$ represents the transfer function between the $j$-th element of the equivalent array and the $i$-th antenna of the TRM which is dependent on the propagation medium. In (3) the only quantities that could not be imposed by the user are these transfer functions. They can be determined in an number of ways, e.g., by vector network analyzer measurements. Thus, once the transfer functions have been determined, we can synthesize the signals received on each TRM antenna as if the wavefront would have been radiated by the electromagnetic equivalent currents distribution, represented by the equivalent array elements, and sampled by the TRM antennas.

As explained in Section II, in the beginning of the timereversal first step there is a temporal window, before a radiated wavefront reaches the cavity walls, where its propagation takes place in free-space conditions. In the second step, as we reversed time, this temporal window will be recreated, but at the end of the excitation duration. During this phase, the time reversal ideally preserves the same directivity that the equivalent array would have originally radiated in free space in the first place. Thus, the role of $\left\{\alpha_{j}\right\}$ is just that of the weights of a phased array and can be computed independently of the propagation medium by means of free-space array design techniques.

Here stands the interest and the novelty of this procedure, there is no more need to sample the field radiated by a source, like in the original use of time-reversal technique, we just need to characterize the propagation medium and then virtually synthesize in real time the signals on the TRM antennas corresponding to any radiation pattern that the equivalent array would be able to generate in free space. Finally, we can reverse these signals in time by applying, in the frequency domain, a phase conjugation of (3) and an inverse Fourier transform in order to obtain them in time domain and feed the TRM antennas with them. Potentially, we are able to do a real-time sweeping of the field direction over an EUT just by changing the synthesized excitation signals on the TRM antennas.

\section{Numerical Results}

To validate this concept of directional wavefront inside the TREC system, numerical simulations have been carried out under a full-wave electromagnetic transient solver using the
Finite Integration Technique as implemented in the software CST's Microwave Studio.

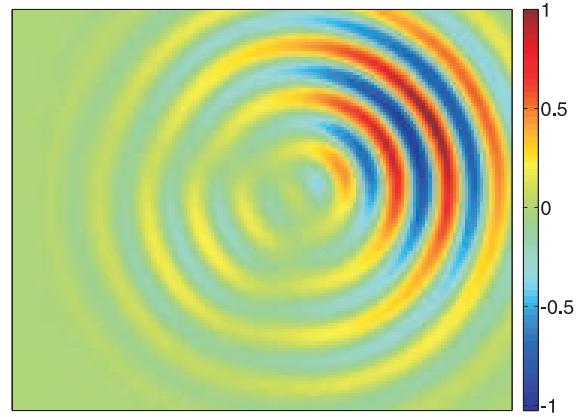

(a)

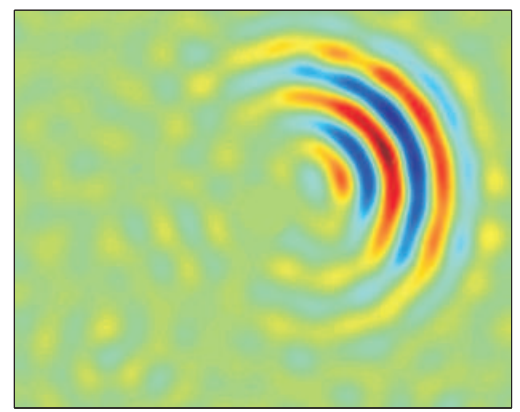

(b)

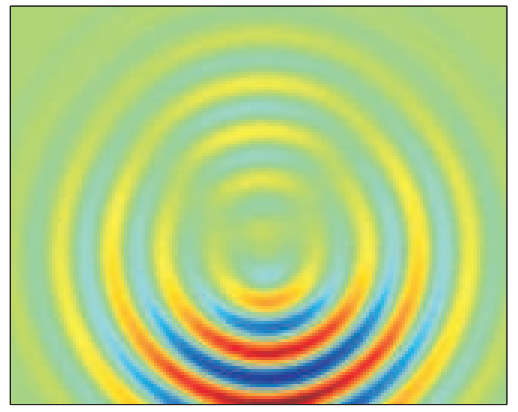

(c)

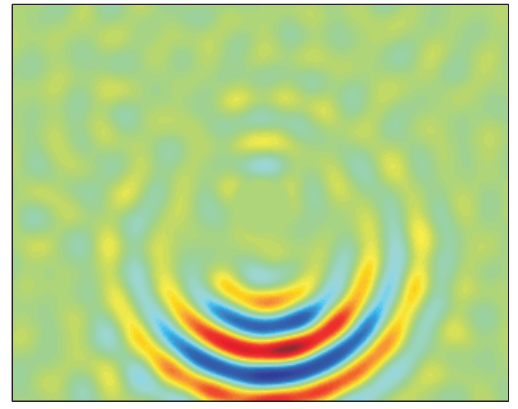

(d)

Fig. 4. Two different directive wavefronts: (a) and (c) are computed with the 2D free-space model; (b) and (d) are TREC numerical simulations.

The cavity modeled has the following electrical dimensions: $l_{a}=16 \lambda_{\mathrm{fc}}, l_{b}=13.2 \lambda_{\mathrm{fc}}, l_{c}=0.6 \lambda_{\mathrm{fc}}$.

The EUT was modeled by a cylindrical shape made out of a perfect conductor with a diameter of $\lambda_{f c}$ and a thickness of 
$\lambda_{\mathrm{fc}} / 2$.

For the excitation signal $\mathrm{x}(t)$, we used a pulse-modulated signal with a magnitude of $1 \mathrm{~V}$, a central frequency of $600 \mathrm{MHz}$ and a bandwidth of $500 \mathrm{MHz}$ which leads to a theoretical modal density of 5.25 modes $/ \mathrm{MHz}$ estimated by Weyl's formula with respect to the cavity dimensions.

The length of the simulation $\Delta T$ was $0.63 \mu \mathrm{s}$, it was limited to this value for computational-time reasons.

The TRM is composed of 8 dipoles of a length of $\lambda_{\mathrm{fc}} / 2$ placed inside the cavity as shown in Figure 1. Attention has been paid to put the antennas at, at least, $0.3 \lambda_{\text {fmin }}$ from the cavity walls, with $\lambda_{\text {fmin }}$ the wavelength associated to the smallest frequency of the excitation signal bandwidth. The exact positions of the TRM antennas, when all the TRM antennas are activated simultaneously, is not a sensitive parameter for the quality of the reconstructed wavefront as shown in [6] but the distance to assure sufficiently uncorrelated antennas is significant as previously said.

Concerning the equivalent array, we placed 3 circular rows of 32 ideal probes around the EUT to prevent coupling between its elements since ideal probes measure directly the field without providing any modification of the propagation medium. As the TREC is a reciprocal system, we injected the excitation signal directly on the TRM antennas, one by one, and collected the signals on the probes rather than the opposite because the simulator does not allow to excite probes. Then, thanks to the linearity of the system, we summed the results by post-processing to synthesize the right TRM signals with (3). In these cases, the weights $\left\{\boldsymbol{\alpha}_{j}\right\}$ were chosen to create a wavefront in a shape of an arc and a length of $1 / 8$ of a circle. Actually, this procedure could be directly transposed, for real measurements, with small opto-electrical probes in order to characterize the transfer functions $\left\{H_{i j}(\omega)\right\}$ previously introduced.

Thus, we generated two different directions for an impinging wavefront, as shown in Figure 4. These figures show a good conformity of the numerical simulations with the theoretical 2D free-space model. The intensity of the field was normalized to its maximum in both cases to be able to compare it qualitatively. For instance the maximum field level, obtained with $20 \mathrm{~mW}$ of injected power, reaches $600 \mathrm{~V} / \mathrm{m}$ just before the impact on the EUT for the TREC simulations, measured by an ideal field probe, while the 2D free-space model generates normalized value of the field. We can observe that the assumption made for the design of the equivalent network array in free space was justified as the TREC system succeeds in creating the desired directions for impinging wavefronts. It is also visible that a "spatial noise" is present around the the coherent wavefront on the numerical results. As it was previously said, the finite number of modes excited in the cavity leads to a degradation of the reconstructed wavefronts.

\section{QuAlity of RECONSTRUCTED WAVEFRONTS}

In order to assess the quality of the reconstructed directional wavefronts we compared the numerical results with the $2 \mathrm{D}$ free-space model of the equivalent array radiation pattern.
For that purpose, we analyzed the distribution of the electric field level inside its background. These values of the "spatial noise", i.e., the incoherent field, were obtained by removing the data corresponding to a disc whose radius covers completely the coherent wavefront. As the wavefront converges towards the EUT, we changed the radius of the disc for different moments to study the evolution of the noise distribution in time. On Figure 5.a, we observe that they remain unchanged, zero-centered, and show a standard deviation of $18 \mathrm{~V} / \mathrm{m}$ which means the SNR $\gamma$ introduced in (2) will change during the time to reach its maximum just before impinging on the EUT because the field magnitude peak increases as the wavefront focuses towards the EUT.

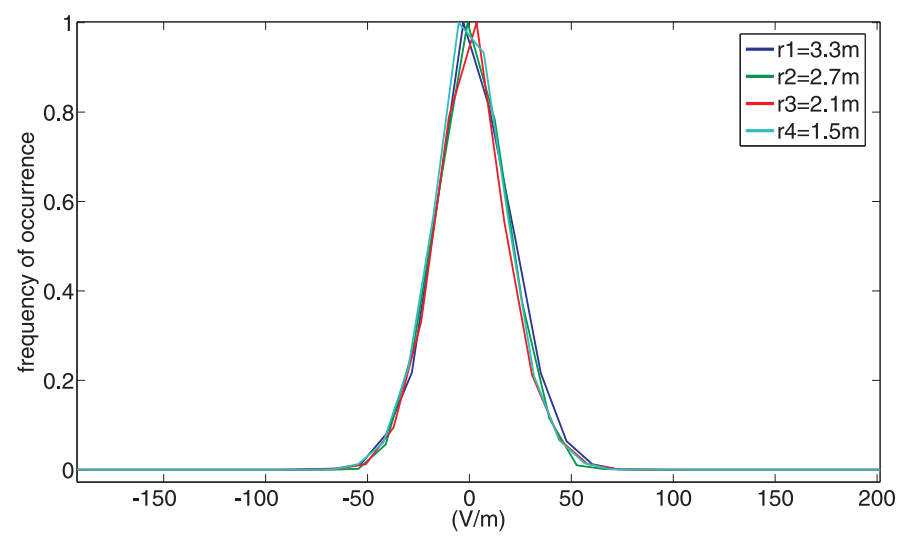

(a)

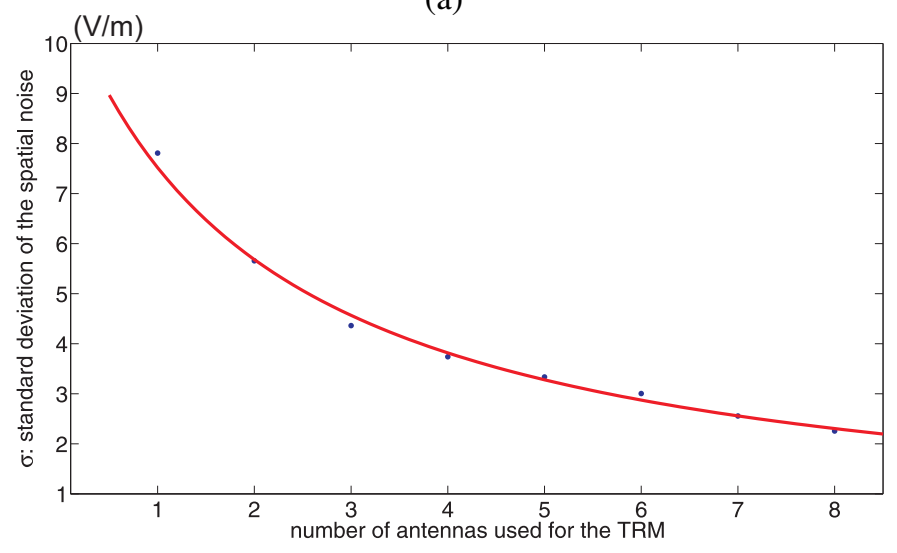

(b)

Fig. 5. "Spatial noise" analysis: (a) frequency of occurrence of the incoherent field values for different radii of removed data, i.e., for different moments of the wave propagation; (b) Evolution of the standard deviation of the "spatial noise" with respect to the number $\mathrm{N}$ of antennas used for the TRM. The solid line is the linear regression in $1 / \mathrm{N}$ of the parameter $1 / \sigma$.

Another aspect studied was the evolution of the standard deviation of the "spatial noise" in respect of the number of antennas for the TRM, presented in Figure 5.b. We can see that the standard deviation decreases as $1 / \mathrm{N}$ with $\mathrm{N}$ the number of antennas. As expected, when the number of antenna increases, more modes are excited so the residual error linked to the reconstructed wavefronts is reduced.

Then, in order to verify if the directivity of the wavefront 
was preserved, we retrieved the value of the field over one circle, placed on the phase center of the wavefront and with a radius covering one of its arcs, represented in the polar diagrams of Figure 6. Once again, the magnitudes were normalized to allow direct comparison.

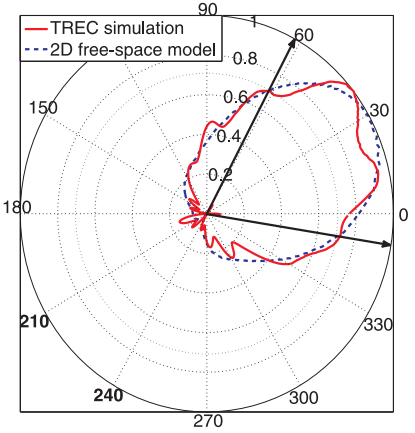

(a)

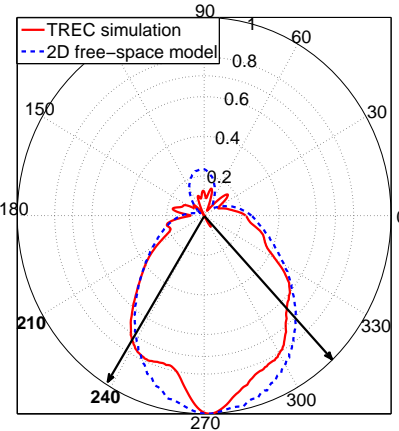

(b)
Fig. 6. Comparison of polar diagrams betweens 2D free-space model and TREC numerical simulations: (a) Polar diagrams for a direction of $25 \mathrm{deg}$; (b) Polar diagrams for a direction of $275 \mathrm{deg}$. The arrows indicate the $3 \mathrm{~dB}$ main lobe. Magnitudes are in a linear scale.

Finally, in order to quantify the quality of the reconstructed directional wavefronts, we focused on the fluctuation of the directivity diagram of the numerical results relative to the 2D free-space reference, over the range of the $3 \mathrm{~dB}$ main lobe angle such as $Q_{3 d B}=\left|E_{\text {freespace } \mid 3 d B}\right| /\left|E_{T R E C \mid 3 d B}\right|$. $\left|E_{\text {freespace } \mid 3 d B}\right|$ and $\left|E_{T R E C \mid 3 d B}\right|$ stand for the normalized field values of, respectively, theoretical model and numerical results. We can see these results, for two directions, in Figure 7, where the mean value of $Q_{3 d B}$ are respectively 0.97 and 0.93 for directions $25 \mathrm{deg}$ and $275 \mathrm{deg}$. At last, we have a rms deviation from the mean of $4.6 \%$ for the first direction and $5.65 \%$ for the second one. The TREC system successfully manages to create deterministic directive wavefronts with a good quality showing residual errors below $6 \%$.

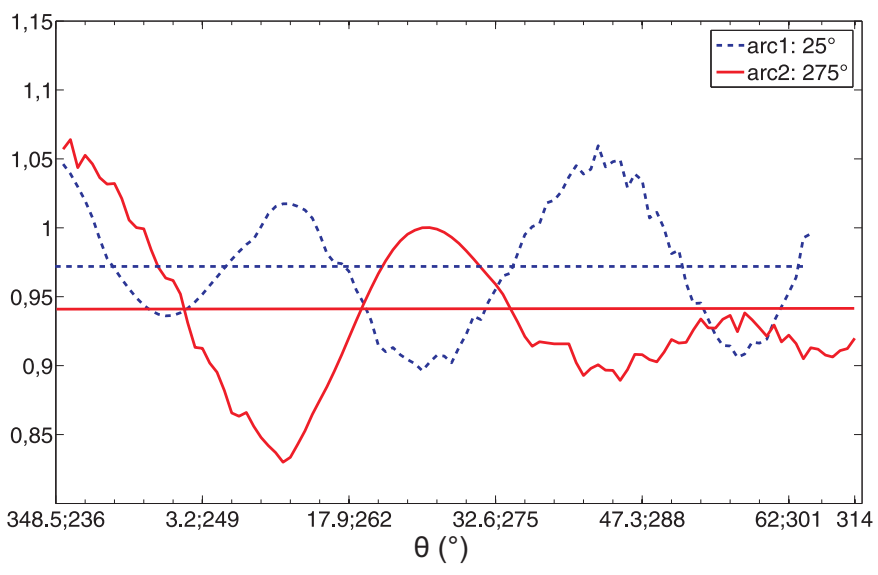

Fig. 7. Quality of the directivity on the $3 \mathrm{~dB}$ main lobe. The abscises values of each $3 \mathrm{~dB}$ main lobe angle for the two wavefronts are separated by a semi-colon. The horizontal lines represent the mean value of $Q_{3 d B}$.

\section{CONCLUSION}

A demonstration of the ability of generating directive deterministic wavefronts inside a 2D Time-Reversal Electromagnetic Chamber has been provided. Numerical results were shown to validate this concept by comparing the simulations to a $2 \mathrm{D}$ free-space model for two different directive radiation patterns. A study of the quality of the reconstructed wavefronts was presented with a good agreement with the free-space model with about $5.7 \%$ percent of rms deviation from the mean value of the field over the $3 \mathrm{~dB}$ main lobe. We expect these results to be even more conclusive with experimental measurements allowing to overcome computational limitations and gain some $10 \mathrm{dBs}$ on the SNR of reconstructed waveforms. Before doing experiments, a validation of this concept in 3D will be done with a new challenge: to extend the properties of the TREC system, generate polarized deterministic wavefronts and propose the TREC as a complementary EMC tool with respect to mode-stirred reverberation chambers and anechoic chambers.

\section{ACKNOWLEDGMENT}

The authors wish to acknowledge the Délégation Générale pour l'Armement (DGA) for supporting this work.

\section{REFERENCES}

[1] D. Hansen, D. Ristau, "Source of problems in the GTEM field structure and recommended solutions," in Proc. IEEE Int. Symp. Electromagnetic Compatibiltiy, pp. 4851, Aug. 1996.

[2] "Radiated, Radio-Frequency, Electromagnetic Field Immunity Test", International Standard, Standard IEC61000-4-3, 1995.

[3] M. L. Crawford, G. H. Koepke, "Design, evaluation and use of a reverberation chamber for performing electromagnetic susceptibility/vulnerability measurements," NBS Tech., vol. Note 1092, no. 4, 1986.

[4] M. Bäckström, J. Loren, O. Lunden, L. Jansson, "Directional properties of microwave coupling for apertures and shielded equipment measured in mode-stirred and anechoic chambers," NIST April 28-May 2, 1997.

[5] L. Jansson, M. Bäckström, "Directivity of equipment and its effect on testing in mode-stirred and anechoic chamber," Proc. IEEE Int. Symp. Electromagnetic Compatibility, pp. 1722, 1999.

[6] H. Moussa, A. Cozza, M. Cauterman, "A novel way of using reverberation chambers through time Reversal," in Proc. ESA Workshop on aerospace EMC, Florence, Italy, March 30- April 1, 2009. Pdf available at: http://hal.archives-ouvertes.fr/docs/00/37/78/67/PDF/ESA09.pdf.

[7] M. Fink, "Time reversal of ultrasonic fields: part I. Basic principles," IEEE Trans. Ultrason. Ferro-electr. Freq. Control, vol. 39, no. 5, pp. 555-566, Sept. 1992.

[8] H. Tortel, G. Micolau, M. Saillard, "Decomposition of the time reversal operator for electromagnetic scattering," Journal of Electromagnetic Waves and Applications, Volume 13, Number 5, 1999 , pp. 687-719(33)

[9] G. Lerosey, J. de Rosny, A. Tourin, A. Derode, G. Montaldo, M. Fink, "Time reversal of electromagnetic waves," American Physical Society, Phys. Rev. Lett., vol. 90, no. 19, pp. 193904, May 2004.

[10] A.E.H. Love, "The integration of the equations of propagation of electric waves," Proceedings of the Royal Society of London, vol. 68, pp. 19-21, 1901.

[11] H.Weyl, "Uber das spektrum der hohlraumstrahlung," Z. Reine Angew. Math., vol. 141, pp. 163181, 1912.

[12] J. R. Wait, "Electromagnetic Radiation from Cylindrical Structures," IEE Electromagnetic waves series 27, 1988.

[13] A Paulraj, "Introduction to space time wireless communication," Cambridge, 2003.

[14] J. de Rosny, "Milieux réverbérants et réversibilité," $P h D$ thesis, Université Paris VI - Pierre et Marie Curie, Oct. 2000. 